\title{
A APLICAÇÃo dos 3R's EM RESíduOS HOSPITALARES: O CASO DO EQUIPO DA BOMBA DE INFUSÃO
}

Sávio Rocha Aleixo (savioaleixo@usp.br)- Escola de Engenharia de São Carlos, Universidade de São Paulo (EESC-USP).

Jéssica dos Santos Leite Gonella (jessica_gonella@hotmail.com) - Universidade Federal de São Carlos (UFSCar).

Janaina Mascarenhas Hornos da Costa (jana.mascarenhas@usp.br) - - Escola de Engenharia de São Carlos, Universidade de São Paulo (EESC-USP).

\section{RESUMO}

A bomba de infusão é um dispositivo eletromecânico utilizada para gerar fluxo de um determinado fluido a pressões superiores a pressão sanguínea superior no local da infusão. No entanto, o uso das bombas de infusão resulta no consumo de materiais hospitalares, descarte devido contaminação ou uso inadequado, ocasionando a geração de resíduos hospitalares, como os equipos, que é um dispositivo plástico responsável pelo transporte de líquidos e fármacos a pacientes. Diferentemente em países desenvolvidos, onde as práticas de redução, reúso e reciclagem dos resíduos hospitalares são evidentes. Para identificar a aplicação dos princípios dos 3R's (reduzir, reusar e reciclar) e política utilizada para a gestão dos resíduos gerados pelas bombas de infusão, os equipos, utilizou-se um protocolo de coleta de dados e entrevistas em hospitais e clínicas da região do interior do estado de São Paulo. Através da pesquisa, foi possível identificar a aplicabilidade parcial dos princípios dos 3R's, políticas de gestão dos resíduos hospitalares das unidades e as percepções dos colaboradores frente aos princípios dos 3'Rs e gestão dos resíduos gerados pelas bombas de infusão nos hospitais e clínicas.

Palavras chaves: bomba de infusão; 3Rs; residuo hospitalar; equipo; economia circular. 


\section{INTRODUÇÃO}

Responsável pela oxigenação, nutrientes, remoção de dióxido de carbono e secreções do corpo humano, o sistema circulatório realiza o transporte de cada elemento a fim de suprir as necessidades humanas, levando em consideração que o sangue em um adulto leva sessenta segundos para completar um ciclo através das vias circulatórias. A bomba de infusão é um dispositivo eletromecânico desenvolvido entre as décadas de 60 e 70 e possui a capacidade de gerar fluxo de um determinado fluido a pressões superiores à pressão sanguínea e são usados para promover o fluxo dos fluidos e fármacos com os equipos (BUTTON, 2018), um agente intermediador da bomba de infusão, através de um sistema de infusão até o paciente. Em relação aos números de equipos, podem chegar a uns vinte tipos com diferentes funcionalidades e cada tipo possui em média dez fabricantes (JANUÁRIO, 2001) e os serviços de enfermagem utilizam a maior parte dos materiais hospitalares e os colaboradores deste setor estão aptos a opinar sobre os mesmos (MAEDA et al. 1991).

No entanto, mesmo aptos para opinar sobre o uso dos equipos e a geração dos mesmos devido à variedade, $58 \%$ dos profissionais e colaboradores da área hospitalar, desconhecem o que são resíduos sólidos de saúde ou conhecem sobre o assunto, mas não possuem a capacidade de explicar a relevância do descarte e manuseio (NAIME; RAMALHO; NAIME, 2007). Por outro lado, quanto a gestão dos resíduos hospitalares, segundo a (ASSOCIAÇÃO BRASILEIRA DE EMPRESAS DE LIMPEZA PÚBLICA E RESÍDUOS PÚBLICOS ABRELPE, 2012), 37,4 \% dos resíduos são incinerados, 21, 7\% seguem para aterros sanitários, 16, 6\% sofrem o processo de autoclave, 13,3\% são descartados em lixões, 5.8 \% são descartados em valas assépticas e apenas 5,2\% são submetidos a micro-ondas. Ainda assim, há escassez de enfermeiros especializados na área de higiene hospitalar e ausência de investimento para atenuar os problemas ocasionados pela geração de resíduos hospitalares em cerca de $90 \%$ dos hospitais brasileiros e apenas $10 \%$ das unidades apresentam profissionais habilitados para a área (BELEI; TAVARES; PAIVA, 1999). 


\section{REVISÃO TEÓRICA}

\subsection{Bomba, Sistema de infusão e equipo}

A bomba de infusão é um dispositivo eletromecânico com a capacidade de gerar fluxo de um dado fluido a pressões superiores à pressão sanguínea na região da infusão (BUTTON, 2018). A figura 1 apresenta a linha do tempo da bomba de infusão. A finalidade geral dos dispositivos de infusão ambulatorial (Figura 1), é introduzir no sistema circulatório de pacientes, líquidos e agentes farmacológicos através de rotas intravenosas, como drogas vasoativas, anestésicos, quimioterápicos, insulina, supressores de dor e hormônios (BUTTON, 2018).

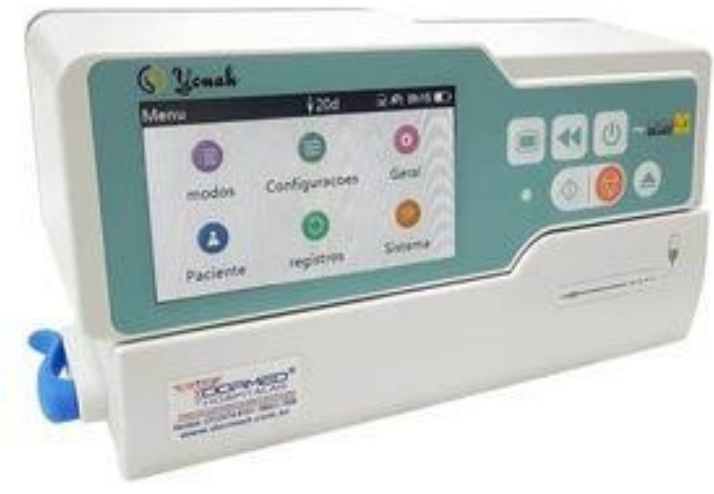

Figura 1: Bomba de infusão Yonah, modelo comercial. Fonte: Dormed (2019). ${ }^{1}$

1 Disponível em: Bomba Infusão para Equipo Universal Yonah - Cmos Drake | Dormed - dormed 
Acoplada a bomba de infusão, encontra-se o equipo, um dispositivo que representa em média 35\% dos equipamentos plásticos de policloreto de polivinila (PVC), utilizados na área médica (Figura 2), para transportar líquido do reservatório para o paciente, (ABNT, 2015).

Figura 2: Equipo universal Yonah, modelo comercial. Fonte: Dormed (2019). ${ }^{2}$

2 Disponível em: Bomba Infusão para Equipo Universal Yonah - Cmos Drake $\mid$ Dormed - dormed 


\subsection{Gestão de material médico hospitalar e resíduos hospitalares}

Segundo Garcia et al., (2012) é denominado gestão de materiais o processo de planejamento, execução e controle, em condições eficientes e econômicas o fluxo de materiais originando das especificações dos artigos a aquisição até a entrega do produto. A atuação do enfermeiro na gestão dos materiais constitui na tomada de decisão, enfatizando a relevância do seu papel na área técnico-administrativa independente ao processo de cuidar e gerenciar. Entretanto o processo de gestão de suprimento de material médico-hospitalar (Figura 3) não possui um tratamento profissional devido, desvalorizando o setor e consequentemente a unidade hospitalar ou clínica (GARCIA et al., 2012).

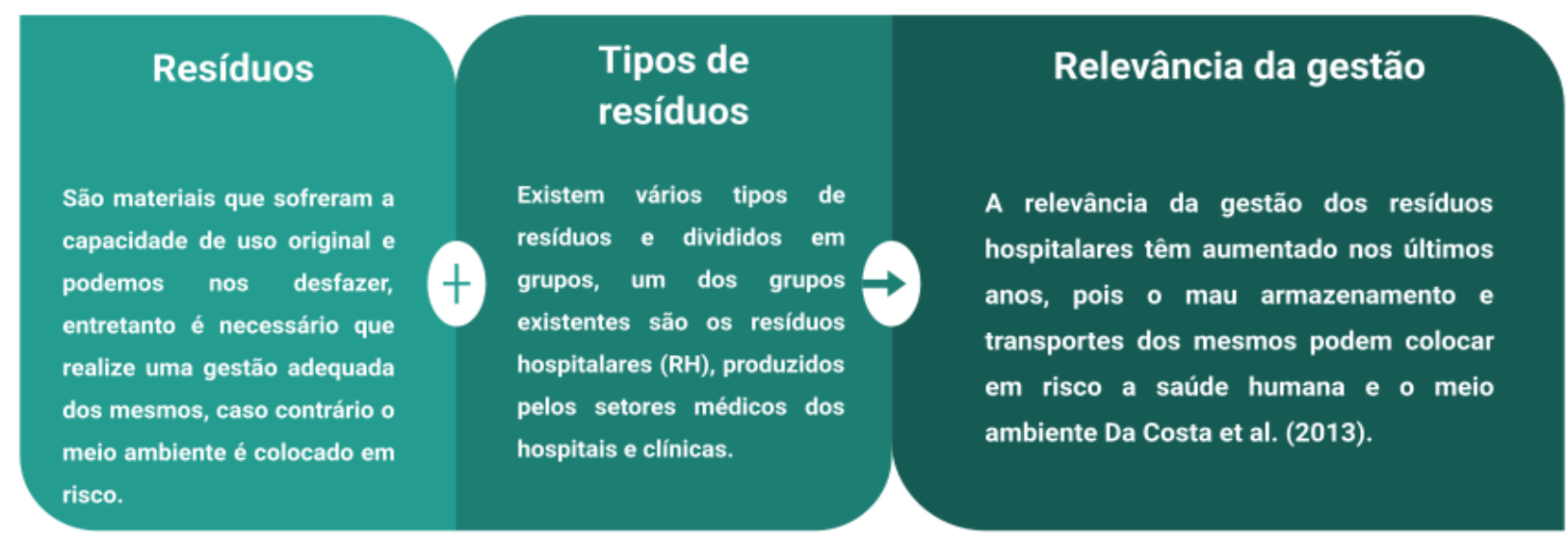

Figura 3: Resíduos e gestão. Fonte: Costa et al. (2013). 
Usualmente, resíduos sólidos e lixo é tudo aquilo que não possui mais utilidade, enquanto resíduo é mais usado para designar sobra (refugo) do beneficiamento de produtos industrializados, os resíduos sólidos são caracterizados como: resíduos nos estados sólidos e semissólidos que derivam das atividades de origem industrial, doméstica, hospitalar, comercial, agrícola e de serviços de varrição (CONSELHO NACIONAL DO MEIO AMBIENTE - CONAMA, 1993). Segundo Agência Nacional de Vigilância Sanitária ANVISA (2006), os resíduos hospitalares (Figura 4) são classificados como resíduos de serviços de saúde e pertencem ao grupo II.

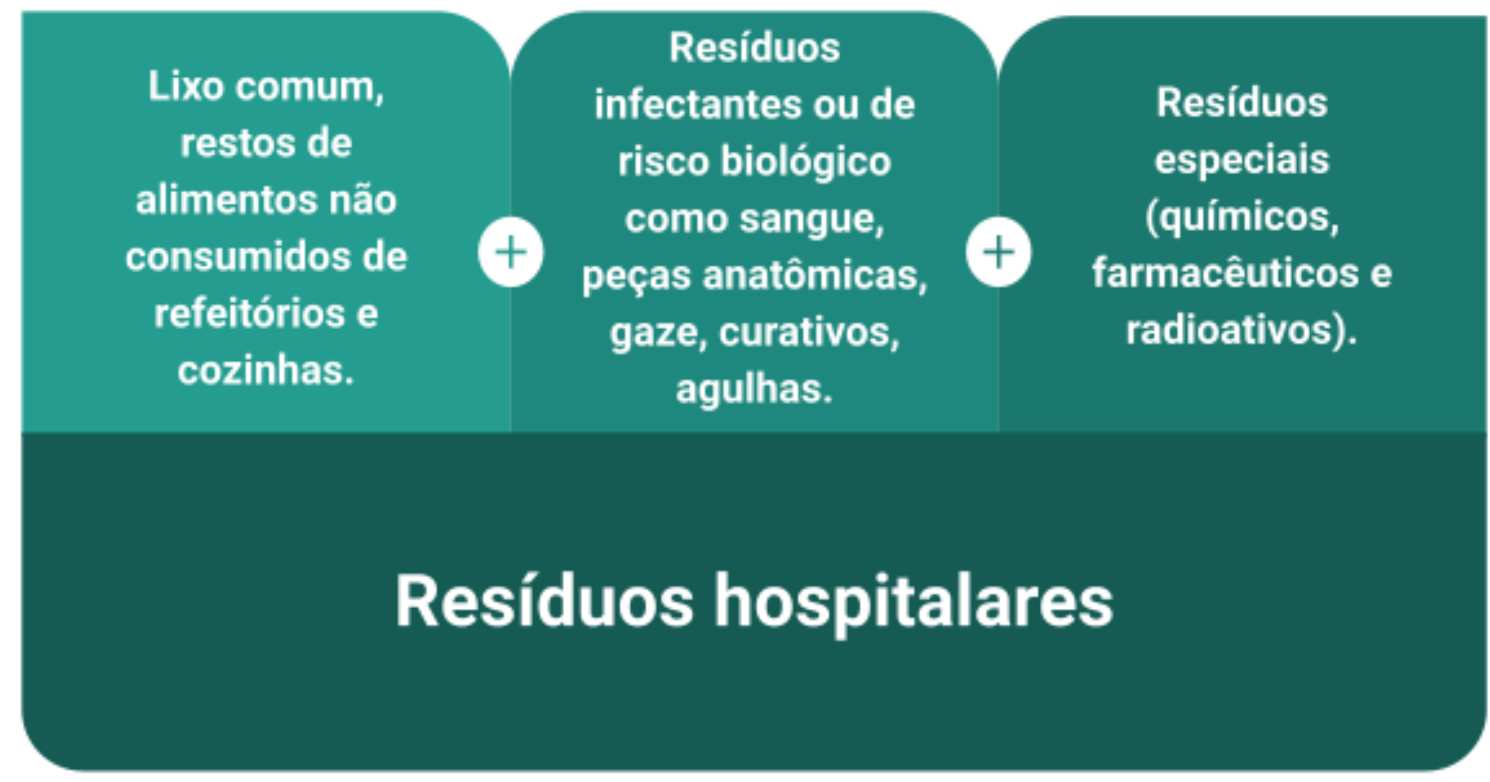

Figura 4: Resíduos hospitalares. Fonte: Ferreira (1995). 
Os resíduos de serviços de saúde são quaisquer serviços realizados de natureza médico-assistencial humana ou animal, clínicas odontológicas, veterinárias, farmácias, centros de pesquisa - farmacologia e saúde, medicamentos vencidos, medicina legal e barreiras sanitárias (ANVISA, 2006). A RDC (Resolução da Diretoria Colegiada) $\mathrm{n}^{\mathrm{o}} 222$ de 28 de março de 2018, regulamenta as boas práticas de gerenciamento dos resíduos de serviços de saúde e classifica o grupo D (Figura 5), os resíduos que não apresentam risco biológico, químico ou radiológico à saúde ou meio ambiente, podendo ser igualados aos resíduos domiciliares e outros similares não classificados como grupo A1.

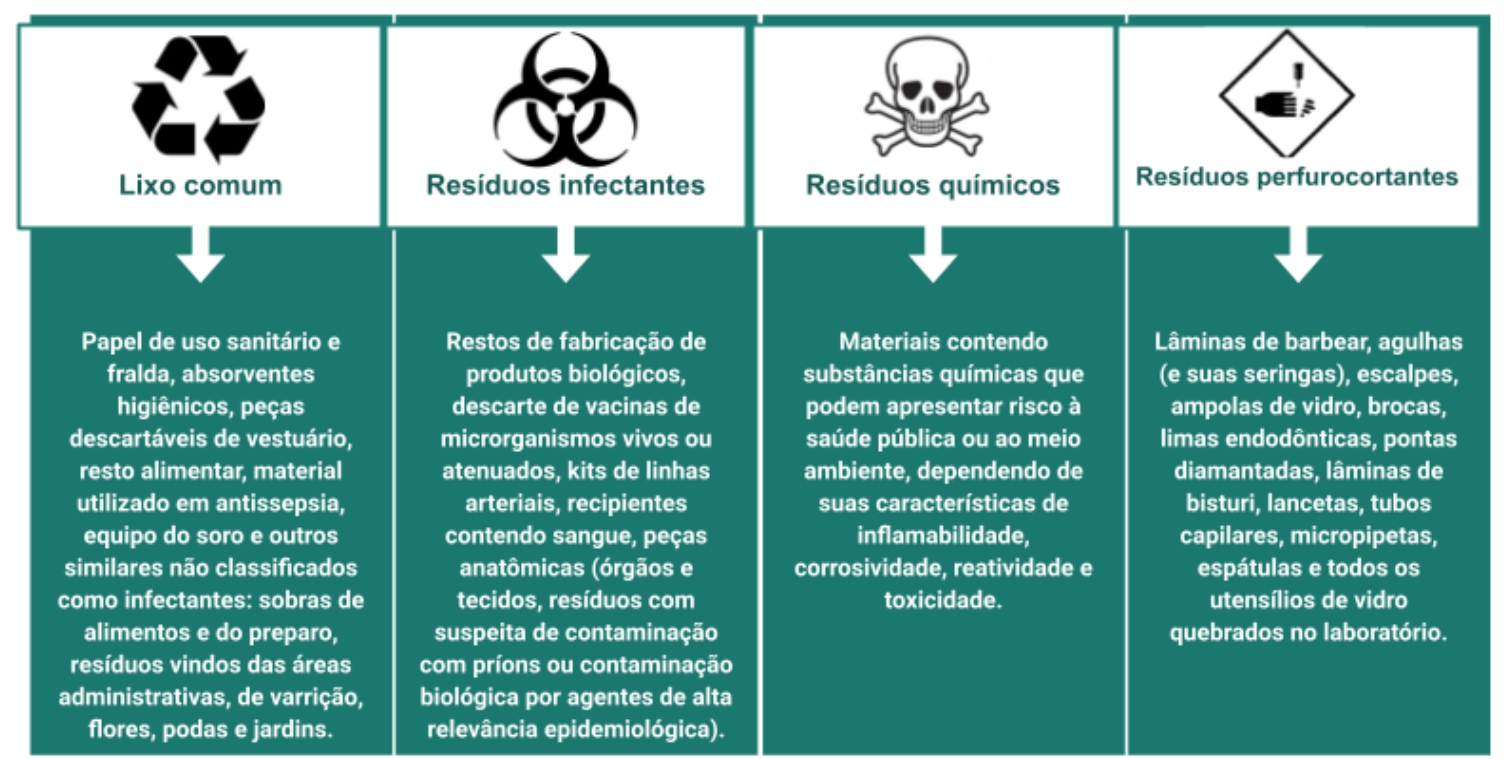

Figura 5: Grupo D - Tipo de resíduos. Fonte: ANVISA (2018). 


\subsection{Sustentabilidade e a aplicação dos princípios dos 3R's}

Os problemas ambientais como poluição da água, aquecimento global, e o uso excessivo da terra, têm colocado em risco a biodiversidade e agravado os danos ao meio ambiente e a saúde das pessoas (CHERTOW, 2001; LIEDER; RASHID, 2016), além disso as atividades ambientalmente nocivas estão se tornando superior à capacidade de absorção pela biosfera, como comprovado pela recorrente crise de resíduos enfrentada pelos países em desenvolvimento (PRESTON; LEHNE, 2017; STINDT; SAHAMINE, 2014).

As abordagens de sustentabilidade emergem com o objetivo de minimizar os impactos ambientais Brundtland (1987), afim de suprir necessidades do presente sem comprometer as futuras gerações, ampara pelo tripé de desenvolvimento ambiental, social e econômico (WISE, 2016). Considerada como uma fronteira da sustentabilidade, a economia circular, representa um modelo de reestruturação das formas de consumo dos recursos naturais, por meio do redesenho dos produtos, introdução de matérias primas biodegradáveis, reaproveitamento de materiais e reciclagem (MOKTADIR et al., 2018).

O conceito foi amparado por escolas de pensamento, como "regenerative design", "industrial ecology" e " cradle to cradle", difundidas nos anos 70, visando incentivar padrões de produção de "fechamento de ciclo" e propondo uma mudança sistêmica que gera oportunidades econômicas, ambientais e sociais Kircherr et al. (2017). Além disso, após 2010, a Fundação Ellen MacArtur dissemina os tópicos de economia circular com o objetivo de torná-la uma referência global do pensamento. Uma estratégia inserida no âmbito da Economia Circular é a aplicação dos 3R's, que se referem às práticas de redução, reúso e reciclagem (JABBOUR et al., 2018), onde os ciclos biológicos e bioquímicos funcionarão segundo o seu fluxo natural por meio da recuperação dos resíduos na cadeia de suprimentos, sendo regidos por três princípios básicos (Figura 6). 


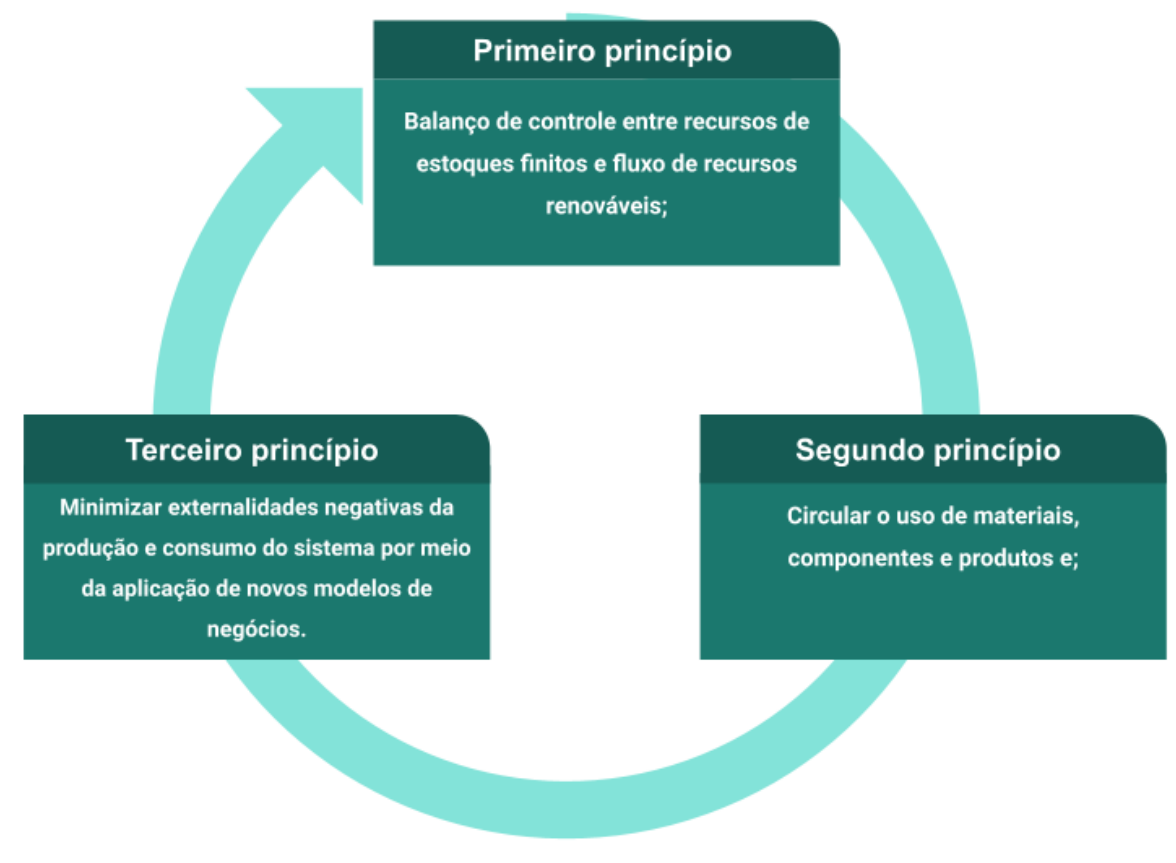

Figura 6: Princípios básicos da recuperação dos resíduos na cadeia de suprimentos.

Fonte: Sthael (2016).

Considerando que a Economia Circular refere-se à produção industrial de sistemas restaurativos e regenerativos, onde produtos e materiais são mantidos no mercado em sua maior utilidade e valor a longo prazo, Batista et al. (2018) sugerem que sua aplicabilidade pode focar nos níveis do produto, nível da organização e nível da indústria (Figura 7). 


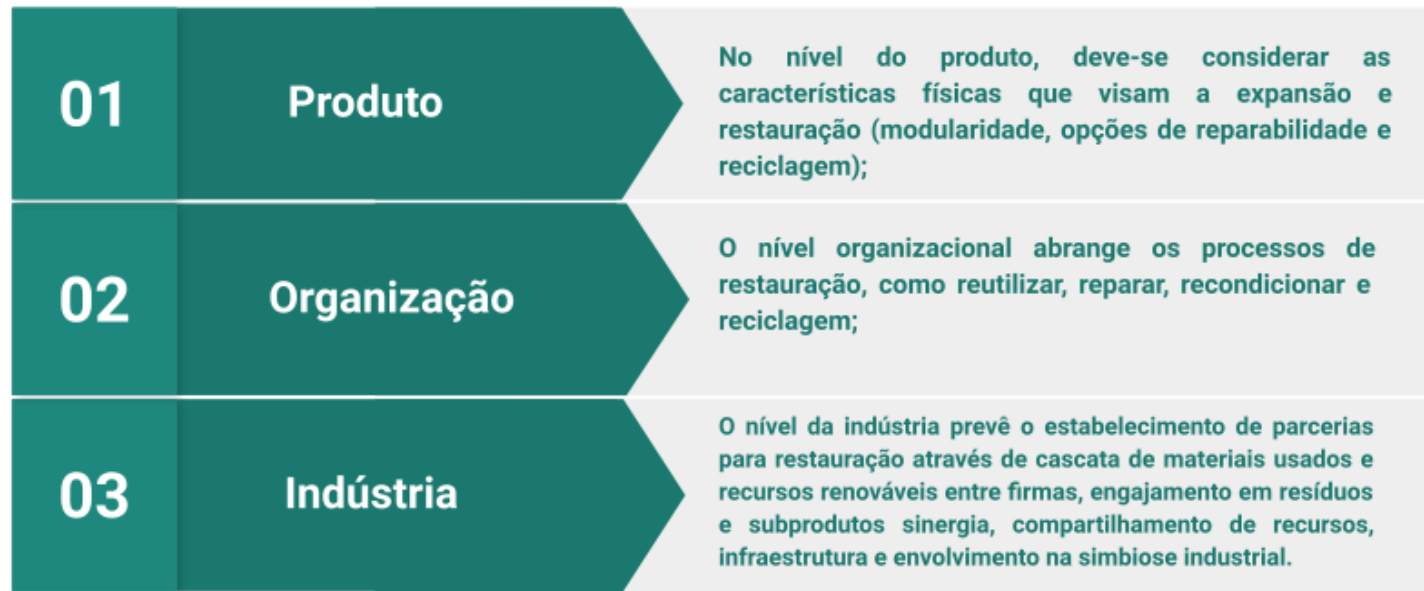

Figura 7: Aplicabilidade da Economia Circular nos níveis do produto, organização e indústria. Fonte: Batista et al. (2018).

Diante disso, nota-se que a aplicação dos 3R's pode-se dar na três diferentes níveis de aplicação, a depender do setor de atuação, produto e cadeia de suprimentos de inserção.

\section{METODOLOGIA}

Para atingir o objetivo será empregada a abordagem qualitativa, que permite captar traços interpretativos do fenômeno estudado CRESWELL (2014) e oferece condições para uma investigação ampla, abarcando o levantamento bibliográfico e documental para a revisão da literatura GIL (2014). Quanto a abordagem descritiva, têm-se a possibilidade de identificar relações, diagnosticando a natureza desta interação CERVO; BERVIAN ; SILVA (2007). O fluxograma (Figura 8) apresenta as fases da pesquisa. 


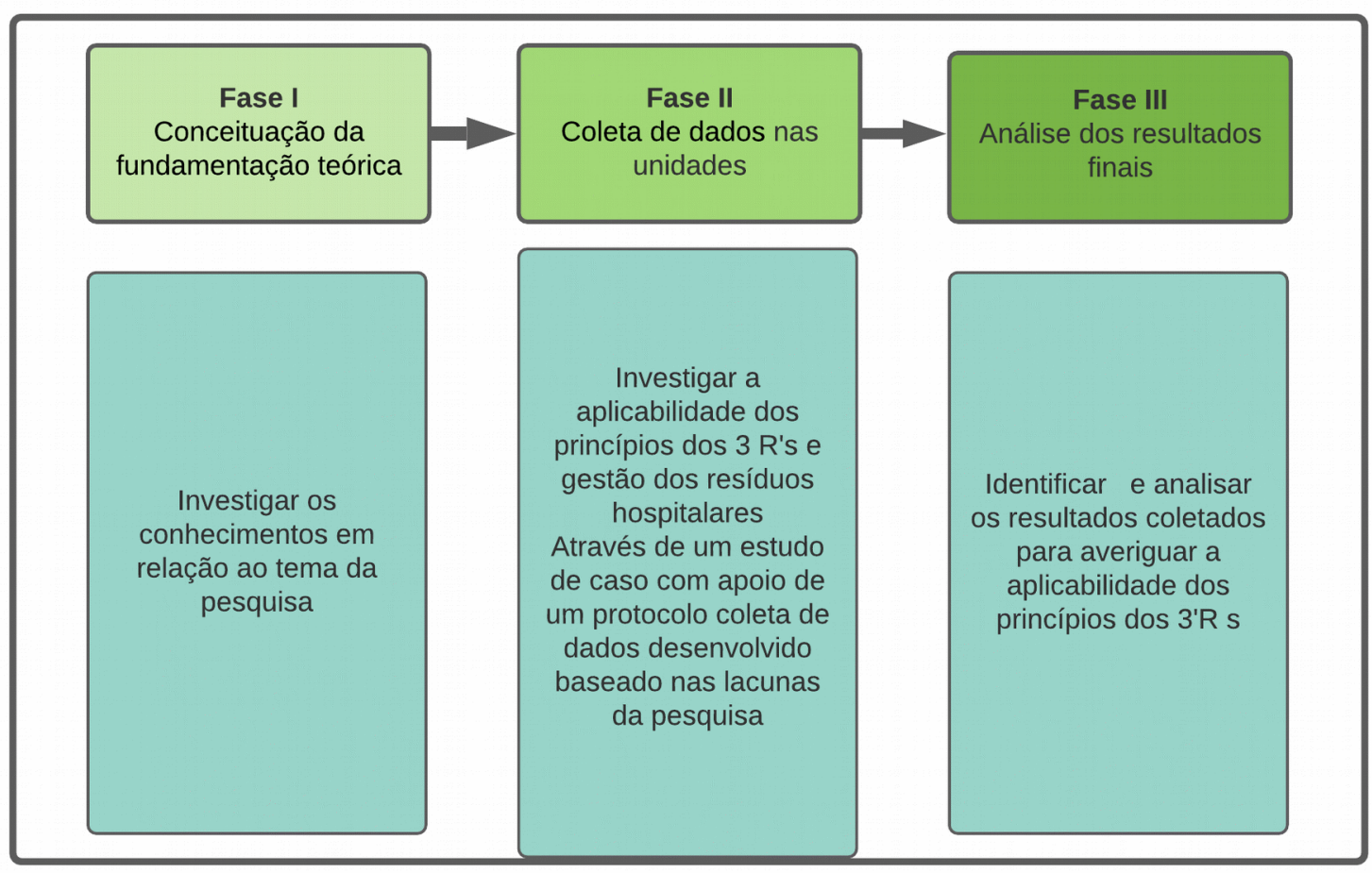

Figura 8: Fases da pesquisa Fonte: Elaborado pelos autores (2018).

Para contemplar o estudo de um fenômeno atual, trata-se de um estudo de caso YIN (2010). O estudo de caso se caracteriza pela manutenção dos traços holísticos do ambiente, além de preservar as transformações ocorridas ao longo do tempo, explorando com intensidade as variáveis de análise (CRESWELL, 2014; YIN, 2010).

A definição da estrutura conceitual teórica foi realizada nas bases científicas Scopus, Web of Science, Ebsco e Google Acadêmico. Além disso, buscou-se dados referente às normas técnicas que regem a utilização dos equipamentos, como as bombas de infusão, materiais médicos hospitalares e equipos. Esta fase facilitou a compreensão dos conceitos e prestou suporte teórico a pesquisa à fase da pesquisa de campo. 
O planejamento dos casos envolveu as unidades hospitalares em São Carlos, Ribeirão Preto, Campinas, Tupã, no interior de São Paulo, onde a apresentação do objetivo da pesquisa e o convite, posteriormente, foi formalizado por e-mail para os responsáveis dos hospitais. Verificou se os colaboradores envolvidos estão cientes acerca da política e da gestão dos resíduos gerados pelas bombas de infusão, bem assim como o descarte e aplicação do princípio dos 3R's (Reduzir, reusar, reciclar). A validação semântica construtos foi realizada por duas especialistas no setor de gestão de resíduos para a correção e ajustamento do instrumento de coleta de dados.

Após a formalização do convite às unidades, a observação in loco e aplicação das entrevistas presencialmente propiciou a maximização do grau de confiabilidade entre a unidade de pesquisa e pesquisadores. Os colaboradores dos hospitais e clínicas médicas que operam e manuseiam as bombas de infusão foram escolhidos e realizada uma abordagem presencial com o protocolo de coleta de dados (APÊNDICE A), elaborado a partir das leituras realizadas durante as pesquisas do referencial teórico . Prezando pela integridade dos hospitais e clínicas médicas, foram omitidos nomes a fim de preservar a identidade dos entrevistados, como apresentado no Quadro 1.

\begin{tabular}{|c|c|c|c|c|}
\hline Entrevistado & Função & $\begin{array}{c}\text { Experiência no } \\
\text { manuseio de Bombas } \\
\text { de Infusão }\end{array}$ & $\begin{array}{c}\text { Unidade } \\
\text { hospitalar }\end{array}$ & Município \\
\hline A & Técnico em Enfermagem & 10 anos & \multirow{2}{*}{ Alfa } & \multirow{2}{*}{ Ribeirão Preto } \\
\hline B & Engenheiro Ambiental & Não possui & & \\
\hline C & Enfermeiro & 8 anos & Beta & São Carlos \\
\hline D & Técnico em Enfermagem & 7 anos & \multirow{2}{*}{ Gama } & \multirow{2}{*}{ Campinas } \\
\hline E & Enfermeiro & 10 anos & Delta & Tupã \\
\hline F & Enfermeiro & 8 anos & . &
\end{tabular}

Quadro 1: Sujeitos de Análises das Unidades da Pesquisa. Fonte: Elaborado pelos autores, (2018). 
A aplicação dos questionários ocorreu entre setembro e novembro de 2018 nas unidades hospitalares. Após a abordagem, a análise dos resultados é considerada como a última etapa da condução do estudo de caso. Para tanto, os dados foram organizados para facilitar a visualização de diversas variáveis multifacetadas (MALHOTRA, 2005). Para a análise dos dados qualitativos utilizou-se o método de análise de conteúdo, que interpreta e compara por meio do processamento das informações (BARDIN, 2009).

\section{RESULTADOS E DISCUSSÃO}

Nesta seção discuta-se a identificação da aplicação dos princípios dos 3R's, políticas utilizadas para a gestão dos resíduos gerados pelas bombas de infusão (os equipos) nas unidades hospitalares e percepções dos colaboradores.

No subobjetivo Reduzir (3R's) foram questionados quanto ao período de utilização dos equipos junto aos pacientes e constatou o uso dos mesmos em até $72 \mathrm{~h}$. Em relação ao descarte do equipo sem a utilização, os respondentes afirmaram quando há contaminação ao abrir a embalagem do equipo, defeito de fabricação ou contaminada. A reutilização do equipo pode ocorrer se for o mesmo fármaco, do contrário é realizada a troca do equipo, sendo descartado. No caso de ser realizadas diferentes prescrições de medicamentos para diminuir o número de equipos, os respondentes negaram, pois há casos em que a cada medicamento são utilizados mais equipos e a média de uso são dois equipos por paciente.

Em relação ao subobjetivo Reusar (3R's) verificaram-se os questionamentos acerca da legislação, processo de esterilização e reutilização do equipo, os respondentes desconhecem alguma RDC que impede a esterilização do equipo, entretanto, segundo a ANVISA a RDC n ${ }^{\circ}$ 61, de 03/02/2016, afirma que o extensor para equipo com ou sem dispositivo para administração de medicamentos, equipo para solução enteral e equipo de transfusão que entram em contato direto com sangue e seus hemocomponentes utilizados para coleta, processamento, armazenamento, transporte, separação e transfusão, são proibidos de reusar. 
Os respondentes afirmaram que nunca houve tentativa de esterilização dos equipos e equipamentos correlatos. A ANVISA, através da RE nº 2.605 de 11 de Agosto de 2006 lista produtos médicos enquadrados como de uso único proibidos de ser reprocessados, equipos descartáveis de qualquer natureza exceto as linhas de diálise, de irrigação e aspiração oftalmológicas; equipos para bombas de infusão peristálticas e de seringas e extensores para equipos com ou sem dispositivo para administração de medicamentos. De acordo com Graziano e Poveda (2017), é possível o reúso, porém mediante a critérios bem definidos, transparência, validação e controle, ainda sim há condição de países em desenvolvimento a possibilidade do reúso artigos de uso único. Sobre utilizar os equipos esterilizados ou reciclados, os entrevistados tiveram a percepção de que não usariam dos equipos esterilizados ou reciclados, porém por se tratar de um produto de recomendação de uso único, os respondentes elencaram alguns posicionamentos como o contexto precisa ser amadurecido e que possuam os padrões de qualidade.

Sobre Reciclar (3R's) foram questionados os processos de descarte e tratamento dos equipos pós uso e constatou-se que após a troca dos equipos, os mesmos são segregados em dois tipos de resíduos, o resíduo comum, quando não há contaminação por parte de resíduos sanguíneos e resíduo infectante, quando há contato com resíduos sanguíneos. Seguindo o protocolo do setor. A responsabilidade de treinamento no processo de descarte são oferecidos pela área de Engenharia Ambiental e Educação Continuada. A destinação dos equipos pós uso dá-se através da equipe de limpeza e posteriormente para coleta interna a um depósito final, esses resíduos incinerados ou encaminhado a aterros e depósitos. Ressalta-se que, artigos médicos hospitalares de PVC pós-consumo, observadas algumas restrições como contato com fármacos e sangue, podem facilmente ser reciclados e usados na fabricação de outros produtos, desde que sejam devidamente coletados (IBPCV, 2018). 
A responsabilidade da coleta dos equipos pós uso compete ao hospital, porém há casos da adesão de empresas terceirizadas para coleta. Os principais problemas com o processo de descarte dos equipos são: 1) segregação errônea por parte das equipes de enfermagem nos locais de descarte, 2) os mesmos descuidam da percepção ao descartar os equipos. Sobre o processo de tratamento os equipos pós uso não recebem tratamento, só em casos de equipos utilizados para fins quimioterápicos, os equipos pós uso não sofrem processo de esterilização. Os entrevistados afirmaram que usariam equipos reciclados, contando que mantivesse os padrões exigidos e o conhecimento do processo de reciclagem.

Sobre a sustentabilidade, as unidades hospitalares possuem um plano de gestão de resíduos de serviços de saúde e a existência de um plano de redução de desperdícios em seus setores, além da reutilização e reciclagem de materiais como papel, vidro, alumínio e lixo orgânico. As unidades hospitalares possuem campanhas de conscientização quanto a sustentabilidade através de treinamentos acerca de segregação no início do ano.

\section{CONCLUSÃO}

Com base nos dados, observa-se que os hospitais possuem o discernimento acerca da gestão dos resíduos de saúde, entretanto os colaboradores das unidades, carecem de mais treinamentos em espaços curtos de tempo, devido à alta rotatividade dos profissionais de enfermagem nas unidades hospitalares. O conceito e a aplicação dos 3R's (Reduzir, Reusar e Reciclar) nestas unidades ainda precisam ser amadurecidas, porém a segregação dos resíduos é realizada através de campanhas internas.

A aplicabilidade dos 3R'S nestes resíduos em relação as leis brasileiras, compreende atualmente no contexto $2 \mathrm{R}$ 'S, reduzir e reciclar, visto que no presente momento desta pesquisa a ANVISA, através das RDC'S e manuais, indicam o uso único, descartando o fator reusar, diferentemente do que ocorre em alguns países da Europa, onde foi apresentado na revisão de literatura que o reúso é realizado. 
Jacobs (2008) afirma que há pouca evidência disponível na literatura publicada para avaliar a prática de reutilização de dispositivos médicos de uso único e faltam dados sobre resultados clínicos. Para melhor gestão e adequação das políticas, as unidades hospitalares necessitam: 1) treinamentos trimestrais, 2) maior sinalização e conscientização por parte dos colaboradores na segregação dos resíduos comuns e resíduos contaminantes, pois os mesmos confundem os resíduos, neste caso a gestão clínica em conjunto com a área ambiental 3) implementar o conceito de gamificação para os colaboradores, onde os mesmos sejam incentivados por cumprir treinamentos, 4) desenvolver um estudo de viabilidade técnica e econômica para a realização dos procedimentos de tratamento dos equipos pós uso, e descartar para cooperativas ou unidades de reciclagem (através de parcerias, público e privada), visto que o equipo é feito de PVC, sendo o mesmo realocado novamente para o ciclo produtivo de matéria prima, gerando impactos positivos nos moldes de uma economia circular. 


\section{REFERÊNCIAS}

ASSOCIAÇÃO BRASILEIRA DE NORMAS TÉCNICAS (ABNT). 60601-2-24:2015 Equipamento eletromédico - Parte 2: Prescrições particulares para segurança de bombas e controladores de infusão.

ASSOCIAÇÃO BRASILEIRA DE EMPRESAS DE LIMPEZA PÚBLICA E RESÍDUOS PÚBLICOS (ABRELPE). Panorama dos Resíduos Sólidos no Brasil. 2013. Disponível em: http://www.abrelpe.org.br/Panorama/panorama2013.pdf Acesso em: 17 ago. 2021.

ALVES, M. A. C. Bombas de infusão: operação, funcionalidade e segurança. Florianópolis. 2002. Dissertação (Mestrado em Engenharia Elétrica) - Centro Tecnológico, Universidade Federal de Santa Catarina, Florianópolis, 2002. Disponível em: márcio alexandre de castro alves1.doc (ufsc.br). Acesso em: 3 dez. 2018.

AGÊNCIA NACIONAL DE VIGILÂNCIA SANITÁRIA. Manual de gerenciamento dos resíduos de serviços de saúde. Brasília: ANVISA; Ministério da Saúde, 2006. Disponível em: http://www.anvisa.gov.br/servicosaude/manuais/manual_gerenciamento_residuos.pdf. Acesso em: 10 set. 2018.

AGÊNCIA NACIONAL DE VIGILÂNCIA SANITÁRIA. Resolução - RE n ${ }^{\circ}$ - 2.605, de 11 de agosto de 2006 - Lista de produtos médicos enquadrados como de uso único proibidos de ser reprocessados. Disponível em: http://portal.anvisa.gov.br/documents/33880/2568070/RE_2605_2006.pdf/33f72d67-f8f64c08-97af-f57802937fbf. Acesso em: 3 dez. 2018.

AGÊNCIA NACIONAL DE VIGILÂNCIA SANITÁRIA. Resolução da diretoria colegiada RDC $\mathrm{n}^{\circ} 222$, de 28 de março de 2018 - boas práticas de gerenciamento dos resíduos de serviços de saúde. Disponível em: Ministério da Saúde (saude.gov.br) Acesso em: 3 dez. 2018 
AGÊNCIA NACIONAL DE VIGILÂNCIA SANITÁRIA. Resolução da diretoria colegiada RDC $n^{\circ}$ 61, de 03 de fevereiro de 2016 Disponível em:RESOLUCÃO-RDC No 61, DE 3 DE FEVEREIRO DE 2016 - Imprensa Nacional (in.gov.br) Acesso em: 3 dez. 2018.

BARDIN, L. Análise de conteúdo. Lisboa: Edições 70, 2009.

BATISTA, L.; BOURLAKIS, M.; SMART, P.; MAULL, R. In Search of a circular supply chain archetype - a content-analysis-based literature review. Production Planning \& Control, v. 29, n.6, p.438-451, 2018.

BELEI, R.A.; TAVARES, M.S.; PAIVA, N.S. Lixo e serviços de saúde: uma revisão. Espaço Saúde, v.1, n.1, p.1-22, 1999.

BRUNDTLAND, G.H. Our common future: report of the world commission on environment and development Med. Confl. Surviv., 4 (1) (1987), p. 300, 10.1080/07488008808408783

BUTTON, V. L.S N. Dispositivos de infusão. Disponível em: dispositivos de infusão - pdf free download (docplayer.com.br) Acesso em: 1 set. 2018.

CERVO, A. L.; BERVIAN, P. C.; SILVA, R. Metodologia científica. 6.ed. São Paulo: Pearson, 2007.

CHERTOW, M.R. The IPAT equation and its variants: changing views of technology and environmental impact J. Ind. Ecol., 4 (2001), pp. 13-29.

CRESWELL, J. W. Research design: qualitative, quantitative, and mixed methods approaches. 4.ed. Nebraska: Sage, 2014.

CONSELHO NACIONAL DO MEIO AMBIENTE. Resolução CONAMA no 5 de 1993, gestão de resíduos e produtos perigosos - tratamento. Disponível em: http://www2.mma.gov.br/port/conama/legiabre.cfm?codlegi=130. Acesso em: 1 set. 2018.

COSTA, C; RIBEIRO, C; SEABRA, F; MOREIRA, F; BARBOSA; SILVA, M. Gestão de resíduos hospitalares. Porto: Faculdade de Engenharia; Universidade do Porto, 2012/2013. (Projeto FEUP). 
http://www.paginas.fe.up.pt/ projfeup/bestof/12_13/files/REL_MIEA102_01.PDF. Acesso em: 9 set. 2018.

FERREIRA, J.A. Solid waste and nosocomial waste: an ethical discussion. Cadernos de Saúde Pública, Rio de Janeiro, v.11, n.2, p.314-320, abr.jun. 1995. Disponível em: http://scielosp.org/scielo.php?pid=S0102-311X1995000200015\&script=sci_arttext\&tlng=en. Acesso em: 10 set. 2018.

GARCIA, S.D; HADDAD, M.C.L; DELLAROZA, M.S.G; COSTA, D.B; MIRANDA, J.M. Gestão de material médico-hospitalar e o processo de trabalho em um hospital público. Revista Brasileira de Enfermagem, Brasília, v.65, n.2, p.339-346, mar./abr. 2012. Disponível em: http://www.scielo.br/pdf/reben/v65n2/v65n2a21.pdf. Acesso em: 8 set. 2018. GIL, A. C. Métodos e técnicas de pesquisa social. 6.ed. São Paulo: Atlas, 2014.

GRAZIANO, K.U; POVEDA, V.B. Reuso de materiais de uso Único. São Paulo: USP, 2017. Curso. Disponível em: https://edisciplinas.usp.br/pluginfile.php/4167963/mod_resource/content/1/REUSO\%20DE \%20MATERIAIS\%20DE\%20USO\%20\%C3\%9ANICO-2017.pdf. Acesso em: 3 dez. 2018.

INSTITUTO BRASILEIRO DO PVC, Aplicações em áreas médicas. Disponível em: http:// pvc.org.br/aplicacoes/area-medica Acesso em: 27 nov. 2018.

JANUÁRIO, L.H (2001) "Equipos de infusão endovenosa - critérios de seleção utilizados por enfermeiros em um hospital de ensino de Belo Horizonte". Belo Horizonte, Escola de Enfermagem da Universidade Federal de Minas Gerais. Disponível em: http://www.enf.ufmg.br/pos/defesas/34M.PDF Acesso em: 27 nov. 2018.

JABBOUR, C. J. C.; JABBOUR, A. B. L.; SARKIS, J.; GODINHO FILHO, M. Unlocking the circular economy through new business models based on large-scale data: An integrative framework and research agenda. Technological Forecasting \& Social Change, v., n., p., 2018 . 
JACOBS, P. Economic Analysis of Reprocessing Single-Use Medical Devices: A Systematic Literature Review. Infection Control and Hospital Epidemiology, Vol. 29, No. 4 (April 2008), pp. 297-301 Published by: The University of Chicago Press on behalf of The Society for Healthcare Epidemiology of America. Disponível em: http://www.jstor.org/stable/10.1086/529587 Acesso em: 20 nov. 2018.

KIRCHERR, J., REIKE, D., HEKKERT, M. Conceptualizing the circular economy: An analysis of 114 definitions, Resources, Conservation and Recycling, Volume 127, 2017, Pages 221-232, ISSN 0921-3449, https://doi.org/10.1016/j.resconrec.2017.09.005.

LIEDER, M. and RASHID, A. Towards circular economy implementation: a comprehensive review in context of manufacturing industry J. Clean. Prod., 115 (2016), pp. 36-51.

MAEDA, M; FUKUI, A; TAMAI, S; MIZUMOTO, S; INADA, Y. Continuous local intraarterial infusion of antithrombotic agents for replantation comparison with intravenous infusion, British Journal of Plastic Surgery, Volume 44, Issue 7, 1991, Pages 520-525, ISSN 0007-1226, https://doi.org/10.1016/0007-1226(91)90009-9.

MALHOTRA, N. K. et al. Introdução à pesquisa de marketing. São Paulo: Editora Pearson Prentice Hall.1 $1^{\mathrm{a}}$ edição., 2005.

MOKTADIR, M.A, RAHMAN, T., RAHMAN, M.H., ALI, S.M., PAUL, S.K. Drivers to sustainable manufacturing practices and circular economy: A perspective of leather industries in Bangladesh, Journal of Cleaner Production, Volume 174, 2018, Pages 1366-1380, ISSN 0959-6526, https://doi.org/10.1016/j.jclepro.2017.11.063.

NAIME, R.; RAMALHO, A.H.P.; NAIME, I.S. Avaliação do sistema de gestão dos resíduos sólidos do Hospital de Clínicas de Porto Alegre. Espaço Saúde, v.9, n.1, p.1-17, 2007. Disponível em: $\quad$ https://www.scielo.br/j/rgenf/a/D87KbG8yjmFcDWrZkvfDXMC/? format=pdf\&lang=pt. Acesso em: 15 ago. 2021. 
PRESTON, F.; LEHNE, J.A Wider Circle? The Circular Economy in Developing Countries; Chatham House, The Royal Institute of International Affairs: London, UK, 2017; ISBN 9781 784132569.

STHAEL, W.R., 2016. Circular economy: a new relationship with our goods and materials Technological would save resources and energy and create local jobs. Nature 531 (7595), $435-439$.

STINDT, D. and SAHAMIE, R. Review of research on closed loop supply chain management in the process industry Flex. Serv. Manuf. J., 26 (2014), pp. 268-293.

WISE, N. Outlining triple bottom line contexts in urban tourism regeneration Cities, 53 (2016), pp. 30-34.

YIN, R. K. Estudo de caso: planejamento e métodos. 4a. edição. Porto Alegre: Bookman, 2010 . 
13ํ Congresso Brasileiro de Inovação e Gestão de Desenvolvimento do Produto 08 a 10 de setembro de 2021 - Escola de Engenharia de São Carlos - Universidade de São Paulo

\section{APÊNDICE A}

Nesta seção encontra-se o protocolo de coleta de dados, utilizado para identificar as lacunas da pesquisa.

Tabela 1: Protocolo de coleta de dados.

\begin{tabular}{|c|c|c|c|}
\hline Sub objetivo & Variáveis & ID & Questões de pesquisa \\
\hline \multirow{3}{*}{$\begin{array}{l}\text { Qua lificação do } \\
\text { entre vistado }\end{array}$} & Dado & 1 & Nome \\
\hline & $\begin{array}{l}\text { Experiéncia do } \\
\text { entrevistado }\end{array}$ & 2 & Há quanto tempo você manvseia as bombas de infusâo \\
\hline & Profissāo & 3 & Qual é a sua função \\
\hline \multirow{5}{*}{ Cenário hospitalar } & Marcas das bombas & 4 & Quais empresas de bombas o hospital possui contrato \\
\hline & Número de bombas & 5 & Qual é a quantidade de bombas de infusâo estabelecidas no contrato com a(s) empresas? \\
\hline & \multirow{2}{*}{ Número de equipos } & 6 & Qual é a quantidade minima de equipos estabelecidas no contrato com a(s) empresas? \\
\hline & & 7 & Qual é a quantidade média de equipos utilizados por semana? \\
\hline & Dado & 8 & Quantos leitos possvi a unidade hospitalar? \\
\hline \multirow{5}{*}{ R' - Redurir } & Periodo de uso & 9 & Qual é o periodo de utilizaçâo dos equipos? \\
\hline & $\begin{array}{l}\text { Descarte devido a } \\
\text { erros }\end{array}$ & 10 & O equipo deve ser descartado mesmo sem ser vtilizado? Em que situaçōes? \\
\hline & \multirow{2}{*}{$\begin{array}{c}\text { Utilização do } \\
\text { equipo }\end{array}$} & 11 & O mesmo equipo pode ser utilizado em quais situaçôes? \\
\hline & & 12 & Há casos em que poderiam ser realizadas diferentes prescriçōes medicamentosas para diminvir o número de equipos? \\
\hline & Dados & 13 & Em mádia o paciente utiliza quantos equipos? \\
\hline \multirow{4}{*}{$\mathbf{R}^{\prime}$ - Reusar } & Legislação & 14 & CME - Existe alguma RDC especifica que impede a esterilizaçâo do equipo? \\
\hline & \multirow{2}{*}{$\begin{array}{l}\text { Processo de } \\
\text { esterilização }\end{array}$} & 15 & CME - Houve tentativa de realizar um procedimento de esterilizaçâo? \\
\hline & & 16 & CME - Existe algum equipamento "semelhante" que foi realizado um processo de esterilização? \\
\hline & $\begin{array}{l}\text { Reutilização do } \\
\text { equipo }\end{array}$ & 17 & Como profissional da área você veria algum problema em utilizar os equipos esterilizados e/ou reciclados. \\
\hline \multirow{9}{*}{ R' - Reciclar } & \multirow{6}{*}{ Processo de descarte } & 18 & Quais saio os procedimentos de descarte dos equipos? \\
\hline & & 19 & De quem é a responsabilidade de treinamento sobre o descarte? \\
\hline & & 20 & Qual é a destinação dos equipos das bombas de infusão após o uso? \\
\hline & & 21 & Qual é a destinação dos equipos das bombas de infusảo spós o descarte final? \\
\hline & & 22 & A preparaçâo dos residvos (equipos) para o descarte final è de responsabilidade do hospital ou terceirizado? \\
\hline & & 23 & Quais saio 03 principais problemas com o processo de descarte dos eqvipos? \\
\hline & \multirow{3}{*}{$\begin{array}{l}\text { Processo de } \\
\text { tratamento }\end{array}$} & 24 & $\begin{array}{l}\text { Quan to a tra tamento intemo: os residvos de serviç os de saúde da bomb a de infusấ, os equipos, recebem algum tipo de } \\
\text { tratamento antes da co leta ex tema ou destino fina1? }\end{array}$ \\
\hline & & \begin{tabular}{|l|l|l}
25 \\
\end{tabular} & Quanto a0s equipos, eles saio encaminhados para reciclagem? \\
\hline & & 26 & Vocé se sentiria confortável em utilizar um equipo de bomba de infusáo reciclado? \\
\hline \multirow{4}{*}{ Sustentabilidade } & \multirow{4}{*}{ Geståo de residuos } & 27 & Na sua unidade de trabalho existe um plano de gestão de residvos de serviços de saúde? \\
\hline & & 28 & Existe um plano de redução de desperdicios em sev setor de trabalho? \\
\hline & & 29 & Existe um plano de revtilizaçâo e reciclagem no sev local de trabalho? \\
\hline & & 30 & $\begin{array}{l}\text { A unid ade ho sp italar possui campanhas de con scien tiza ção qu anto a su stentabilida de? Com relação a } 03 \text { resid vos de serviços de } \\
\text { saúde? }\end{array}$ \\
\hline
\end{tabular}

Fonte: Elaborado pelos autores, (2018). 